\title{
StrainsApp: el catálogo de microorganismos españoles accesible "on-line"
}

Lidia Rodrigo-Torres¹, Aurora Zuzuarregui Miró², José Miguel López Coronado³ y Rosa Aznar Novella4

Colección Española de Cultivos Tipo (CECT). ' Gestora REDESMI. ${ }^{2}$ Gestora recursos microbianos CECT.

${ }^{3}$ Responsable de informática y patentes. ${ }^{4}$ Directora de la CECT, REDESMI y MicroBioSpain.

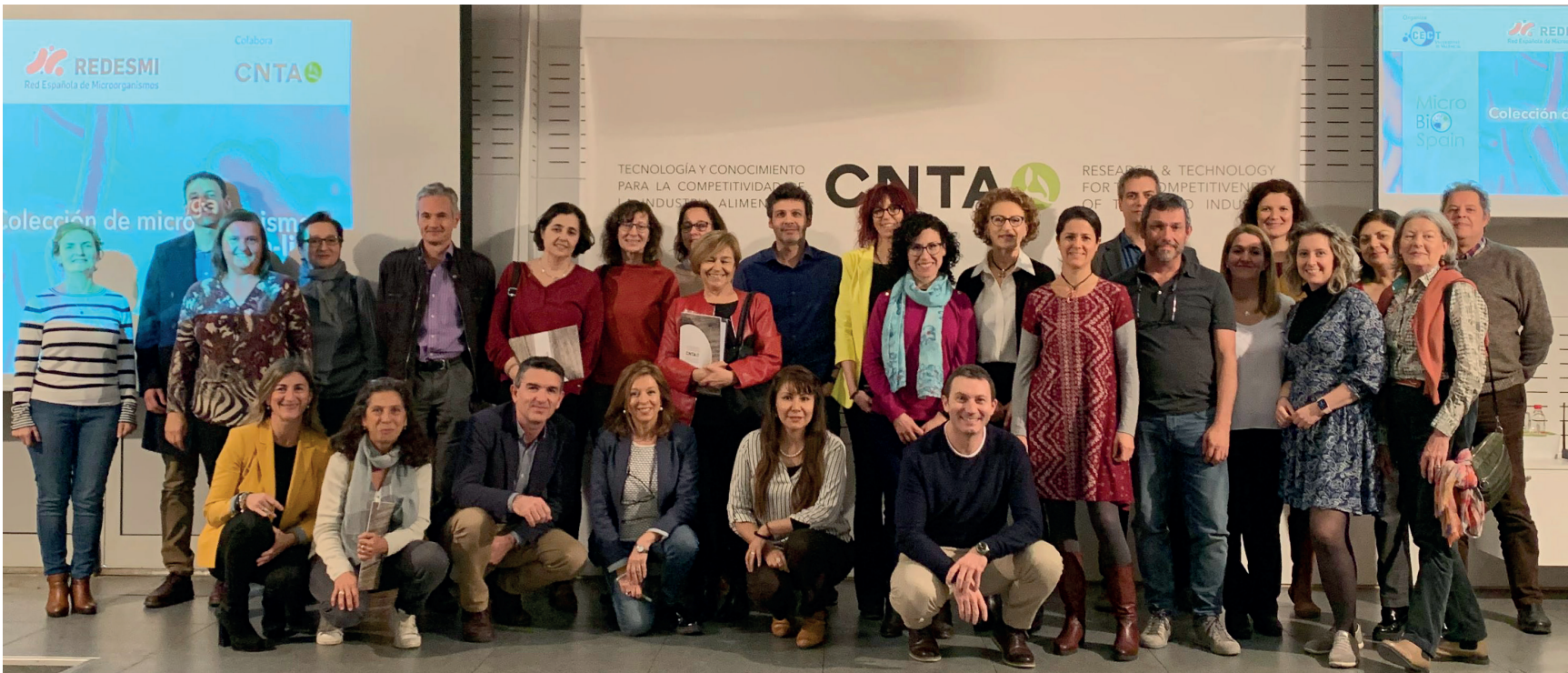

Miembros de REDESMI y MICROBIOSPAIN en la jornada "MICROBIOSPAIN: la colección de microorganismos españoles accesible "on-line", celebrada el pasado 28 de febrero en el CNTA (San Adrián, Navarra)

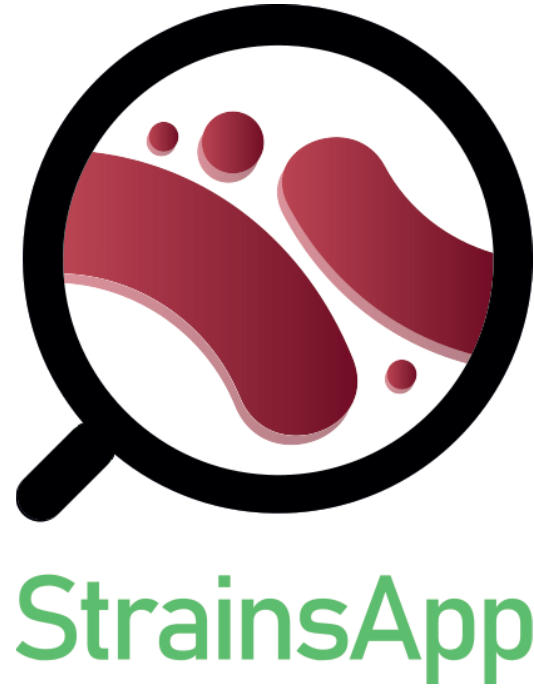

StrainsApp es una aplicación web que ha desarrollado la CECT (Colección Española de Cultivos Tipo) con un doble objetivo: i) facilitar a las colecciones de investigación registradas en REDESMI (Red Española de Microorganismos) la gestión de las cepas que mantienen y ii) permitir la consulta pública on-line de esas cepas, visibilizando y facilitando el acceso a estos recursos microbianos con potencial biotecnológico.

Se trata de una iniciativa que forma parte del proyecto "Conservación sostenible de recursos microbianos españoles bajo estándares de calidad, mediante una aproximación integradora y potenciando su visibilidad", RMP2015-000100-00, financiado por la Agencia Española de Investigación a través del INIA con fondos FEDER. Así mismo, se enmarca en la Red de Excelencia MicroBioSpain CGL2016-81969REDT, financiada por el Ministerio de Ciencia, Innovación y Universidades.

Tanto las colecciones de investigación REDESMI como las colecciones públicas españolas, la CECT y el Banco Español de
Algas (BEA), han participado en la selección y designación de los campos de información que serán comunes en la base de datos pública. Estos campos son el punto de partida para cualquier colección que desee incorporar los datos de sus cepas, a la vez que facilitan una búsqueda más eficiente en las consultas públicas al catálogo. Pero además, el investigador responsable podrá crear los campos particulares que requiera para las cepas de su colección, de manera que se recopile la mayor información posible. Por tanto, StrainsApp es una aplicación que permite a las colecciones que la utilicen personalizar completamente la base de datos con la que gestionar sus cepas.

La utilización de StrainsApp (strainsapp. redesmi.es) para introducir datos sobre las cepas y organizar la propia colección es abierta para los miembros inscritos en REDESMI y requiere de usuario y contraseña. 


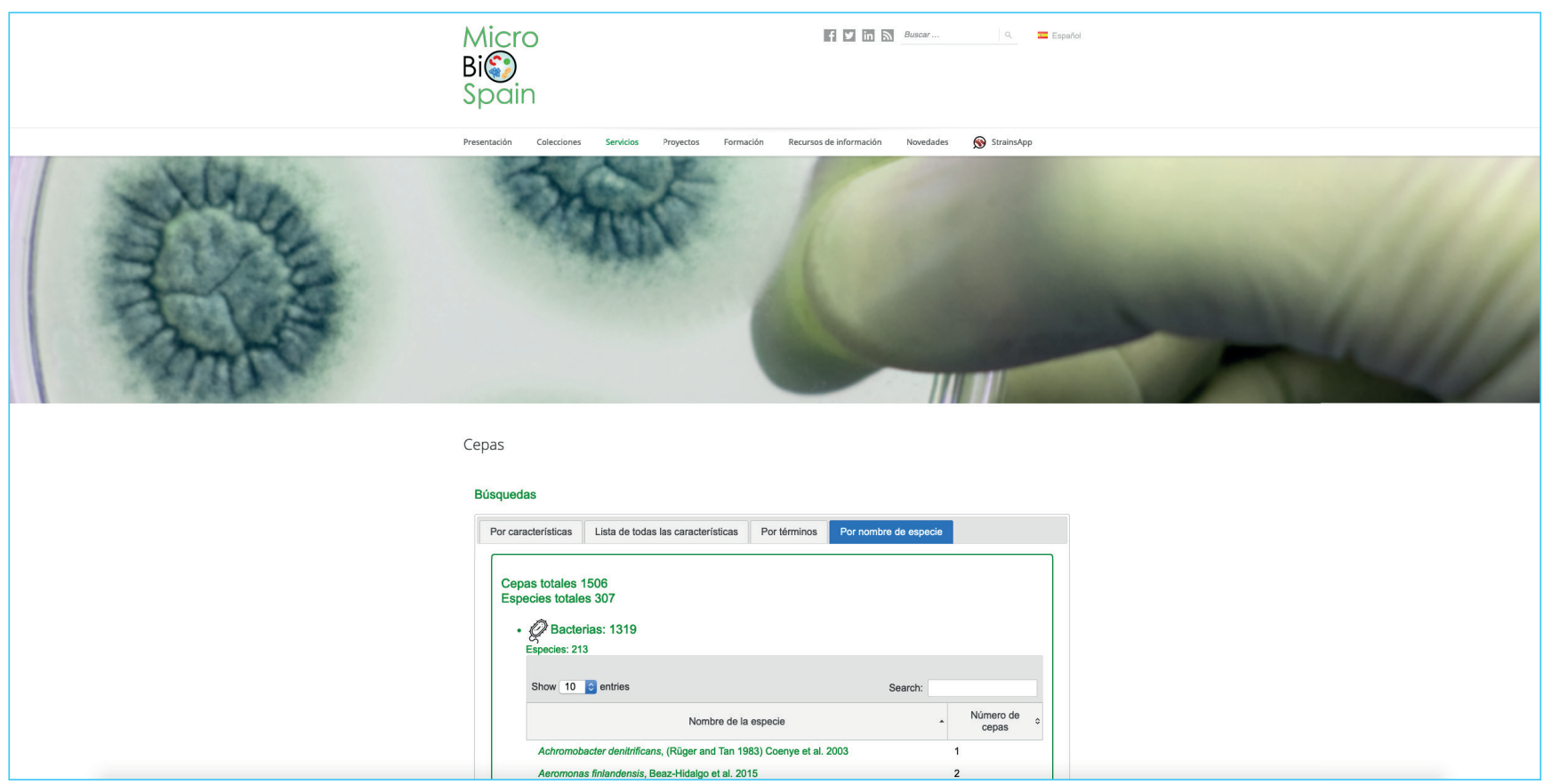

Página de consulta del catálogo de cepas de las colecciones REDESMI a través de la web de MicroBioSpain (www.microbiospain.org/servicios-cepas/)

Los términos de uso de la aplicación han sido consensuados y han de aceptarse para su utilización. Entre otros aspectos, indican que el responsable de la colección puede decidir qué información "relevante en cuanto a aplicación biotecnológica” quiere hacer pública.

La consulta del catálogo de cepas es abierta al público a través de la web de MicroBioSpain (www. microbiospain.org/servicios-cepas/) y el usuario puede contactar con el responsable de la colección, en caso de que una 0 más cepas susciten su interés, bien para establecer colaboración científica, bien para explorar su posible aplicación o explotación comercial. En la fase inicial, el catálogo cuenta con 1.506 cepas de 16 de las 43 colecciones que actualmente forman parte de REDESMI, cuya información sobre el investigador responsable, la institución a la que pertenece, ubicación y descripción de los grupos de microorganismos que alberga, está recogida en la sección de colecciones de la web de MicroBioSpain (www. microbiospain org/colecciones).

Con el apoyo de los proyectos arriba mencionados, la CECT y el CNTA (Centro Nacional de Tecnología Alimentaria) organizaron la jornada "MICROBIOSPAIN: la colección de microorganismos españoles accesible "on-line", celebrada el pasado $28 \mathrm{de}$ febrero en el CNTA (San Adrián, Navarra). Se planteó como una actividad para fomentar la interconexión del tejido empresarial con el científico y promover la explotación comercial de los recursos microbianos españoles, girando en torno al lanzamiento de la aplicación StrainsApp. Tuvo una gran acogida, incluyendo representantes de REDESMI y un importante número de empresas del sector agroalimentario.

Como ponentes participaron por orden de intervención, Daniel Ramón, Vicepresidente de I+D en Nutrición y Salud de Biopolis - ADM, que nos presentó los principales canales, barreras y opciones para el uso de microorganismos en la industria alimentaria; Noelia Vallejo Pedregal, de la Subdirección General de Biodiversidad y Medio Natural (Ministerio para la Transición Ecológica), que nos habló sobre la normativa de acceso y uso de recursos genéticos en el marco del Protocolo Nagoya; Rosa Aznar como IP que presentó los proyectos sobre recursos microbianos que lidera la CECT; José Miguel López Coronado, responsable de informática de la
CECT, que presentó la aplicación StrainsApp, y los miembros de MicroBioSpain (BEA, CECT, CIAL, IATA, INIA, IPLA, IRTA y CNTA) que presentaron los últimos avances en el uso de microorganismos.

Disponer de un catálogo de microorganismos españoles, así como de una web que integra los grupos de investigación que los mantienen, es una de las actuaciones encaminadas a conformar el nodo español de la Infraestructura de Investigación (RI) Microbial Resource Research Infraestructure (MIRRI) del que por el momento forman parte la CECT y el BEA.

A nivel europeo, MIRRI (www.mirri.org) continúa activo a través de los países que hemos firmado el MoU (Memorandum of Understanding) realizando los trámites para constituirse legalmente como ERIC (European Research Infraestructure Consortium) y, a la vez, mediante la participación en los proyectos "EOSC-Life" www.eosc-life.eu (INFRAEOSC-04-2018, Research and Innovation Action (RIA) GA No.: 824087, 01/03/2019 - 28/02/2023) y ismirri21 . mirri.org (INFRADEV-03-2019 RIA GA No.: 871129, 01/02/2020 - 31/01/2023) que facilitarán su implementación. 